\title{
Direct analysis of dispersive wave fields from near-field pressure measurements
}

\author{
Lars Hörchens ${ }^{\text {a) }}$ \\ Laboratory of Acoustical Imaging and Sound Control, Delft University of Technology, P.O. Box 5046, \\ 2600 GA Delft, The Netherlands
}

(Received 4 August 2010; revised 9 July 2011; accepted 27 July 2011)

\begin{abstract}
Flexural waves play a significant role for the radiation of sound from plates. The analysis of flexural wave fields enables the detection of sources and transmission paths in plate-like structures. The measurement of these wave fields can be carried out indirectly by means of near-field acoustic holography, which determines the vibrational wave field from pressure information measured in a plane close to the plate under investigation. The reconstruction of the plate vibration is usually obtained by inverting the forward radiation problem, i.e., by inversion of an integral operator. In this article, it is shown that a pressure measurement taken in the extreme near-field of a vibrating plate can directly be used for the approximate analysis of the dispersive flexural wave field. The inversion step of near-field acoustic holography is not necessarily required if such an approximate solution is sufficient. The proposed method enables fast and simple analysis of dispersion characteristics. Application of dispersion compensation to the measured field allows for visualizations of propagating wavefronts, such that sources and scatterers in the plate can be detected. The capabilities of the described approach are demonstrated on several measurements.

(C) 2011 Acoustical Society of America. [DOI: 10.1121/1.3626164]
\end{abstract}

PACS number(s): 43.60.Sx, 43.40.Dx, 43.20.Tb [JDM]

Pages: 2035-2042

\section{INTRODUCTION}

Dispersive flexural waves play a significant role in the radiation of sound from constructions made of plates or plate-like structures. ${ }^{1}$ The insight gained from their analysis can be used to cope with problems related to structural vibration and sound radiation. The measurement of flexural wave fields can be carried out indirectly by means of planar nearfield acoustic holography (NAH). The vibration of the plate is thereby reconstructed from measurements of pressure ${ }^{2}$ or particle velocity ${ }^{3}$ in a plane close to the vibrating plate. Acoustical holography constitutes an ill-posed inverse problem requiring regularization. ${ }^{4}$ Several authors have proposed additions and improvements in order to obtain accurate reconstructions ${ }^{5}$ and improve the robustness to noise. ${ }^{6}$

However, it can be shown that essential information on the dispersive flexural wave field is contained directly in the pressure measurement taken in the near-field. A useful approximation of the surface normal velocity can be obtained from the pressure measurement by a simple filtering operation, thereby circumventing the solution of an illposed inverse problem. This relatively simple approach is sufficient for a number of applications. For example, the dispersion characteristics of the medium under investigation can be determined. Furthermore, it is possible to visualize the propagation of wavefronts in combination with techniques for dispersion compensation, ${ }^{7}$ thereby enabling the analysis of wave propagation in the plate and the detection of scatterers and reflectors.

\footnotetext{
a) Present address: Applus RTD, Delftweg 144, 3046 NC Rotterdam, The Netherlands. Author to whom correspondence should be addressed. Electronic mail: Lars.Horchens@applusrtd.com
}

Direct measurements on flexural wave fields at single points can be carried out using accelerometers, for instance. For the measurement of an entire wave field, a virtual array, i.e., a system in which a single sensor can easily be repositioned, has to be preferred. Whereas direct measurements of flexural wave fields using laser vibrometry achieve high speed and accuracy, albeit at substantial hardware costs, the usage of a microphone for near-field measurements provides a relatively cheap solution for vibration analysis.

The proposed approach makes use of this basic measurement setup needed for acoustic holography. However, it abandons the associated cumbersome inversion of an integral operator by introducing a simplified point-to-point correspondence between the flexural wave field and a pressure measurement taken in the extreme near-field of the plate. The validity of this approximation is shown to depend on the distance between the plate and the plane in which the pressure measurement is taken.

The theory of near-field acoustic holography is outlined in Sec. II A, followed by the derivation of an approximation for the extreme near-field. The application of the proposed approach to vibration analysis in plates is described in Secs. II B and II C, and followed by the presentation of a method for the removal of the effects of dispersion in Sec. II D. The performance on measurements is tested and demonstrated in Sec. III, followed by discussion and conclusions.

\section{THEORETICAL BACKGROUND}

\section{A. Planar near-field acoustic holography}

The pressure $P(\underline{x}, \omega)$ in a point $\underline{x}=(x, y, z)^{\mathrm{T}}$ at the angular frequency $\omega$ due to a plate vibrating with normal 
velocity $V_{z}\left(\underline{x}_{0}, \omega\right)$ can be calculated using the Rayleigh integral: ${ }^{8}$

$$
P(\underline{x}, \omega)=2 j \omega \rho \int_{S} V_{z}\left(\underline{x}_{0}, \omega\right) G\left(\underline{x}, \underline{x}_{0}, \omega\right) \mathrm{d} S,
$$

with $S$ denoting the plate surface over which the integral is taken, $\rho$ being the density of air, and $G\left(\underline{x}, \underline{x}_{0}, \omega\right)$ being the three-dimensional free-field Green's function for acoustical waves:

$$
G\left(\underline{x}, \underline{x}_{0}, \omega\right)=\frac{e^{-j k\left|\underline{x}-\underline{x}_{0}\right|}}{4 \pi\left|\underline{x}-\underline{x}_{0}\right|},
$$

with $j$ being the imaginary unit $(j=\sqrt{-1})$ and $k$ representing the wavenumber $k=\omega / c, c$ being the velocity of sound in air. The $x$ - and $y$-direction are the in-plane directions. The $z$-direction is perpendicular to the plate, such that $\Delta z=\left|z-z_{0}\right|$ describes the distance between the vibrating plate and the plane in which the measurements are taken. The geometry of this setup is shown in Fig. 1.

The reconstruction of the surface normal velocity $V_{z}\left(\underline{x}_{0}, \omega\right)$ from pressure measurements $P(\underline{x}, \omega)$ requires the inversion of Eq. (1). This equation is a Fredholm integral equation of the first kind describing a spatial convolution with the Green's function. The inverse problem of near-field acoustic holography can, therefore, be interpreted as a deconvolution problem. Its ill-posed nature requires regularization to avoid the excessive amplification of noise. ${ }^{6}$

\section{Approximation in the extreme near-field}

The Rayleigh integral given by Eq. (1) states that all points in the source plane have influence on all points in the measurement plane. However, this influence is weighted by the amplitude of the Green's function. Eq. (2) shows that this amplitude depends inversely on the distance $d$ between a point in the source plane and the measurement plane.

$$
\left|G\left(\underline{x}, \underline{x}_{0}, \omega\right)\right| \propto \frac{1}{d}, \quad \text { with } d=\left|\underline{x}-\underline{x}_{0}\right| .
$$

By taking the distance $\Delta z$ between the plate and the measurement plane as a parameter, the influence of a point on the vibrating plate on a point in the measurement plane

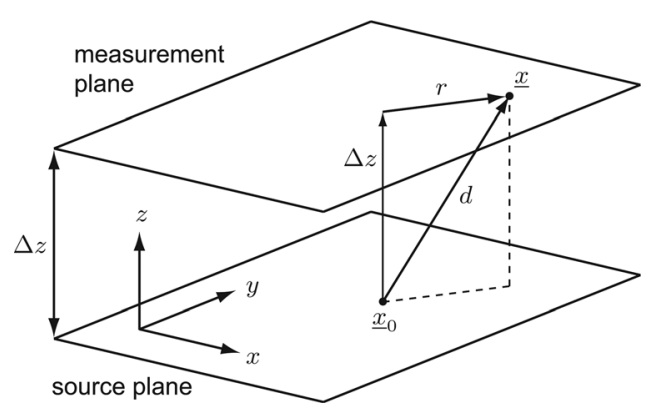

FIG. 1. Geometry used for the description of near-field acoustic holography. can be expressed in terms of the in-plane distance $r=\sqrt{\left(x-x_{0}\right)^{2}+\left(y-y_{0}\right)^{2}}$ as shown in Fig. 1:

$$
\left|G\left(\underline{x}, \underline{x}_{0}, \omega\right)\right| \propto \frac{1}{\sqrt{r^{2}+(\Delta z)^{2}}} .
$$

Figure 2 presents a plot of this function for different distances $\Delta z$ between the source plane and the measurement plane.

As expected, the closest point on the vibrating plate has the strongest influence on the measurement. It can be seen that the influence of neighboring points decays rapidly with increasing in-plane distance $r$. This is especially the case if the measurement is taken in the extreme near-field, i.e., at close proximity to the vibrating plate. As an example, a grid of $1 \mathrm{~cm}$ spacing for measurement and reconstruction is considered. If the measurement is taken at a distance of $3 \mathrm{~mm}$ from the plate, for instance, the influence of a neighboring point is found to be more than $10 \mathrm{~dB}$ lower than the influence of the point on the plate which is closest to the microphone.

The extreme near-field can be defined by requiring a certain quotient $0<Q<1$ describing the relative influence of neighboring measurement points with distance $r$. The maximal measurement distance $\Delta z_{\max }$ can then be derived from Eq. (4):

$$
\Delta z_{\max }=\frac{r Q}{\sqrt{1-Q^{2}}} .
$$

If the minimal difference in influence between neighboring points is chosen to be $6 \mathrm{~dB}$, i.e., $Q=0.5$, Eq. (5) can be used to derive that the maximum distance $\Delta z_{\max }$ for measurements is approximately $5.8 \mathrm{~mm}$ for a grid spacing of $r=1 \mathrm{~cm}$.

Hence, in rough approximation, the contribution of the surrounding points can be neglected in the extreme nearfield. Equation (1) is then simplified:

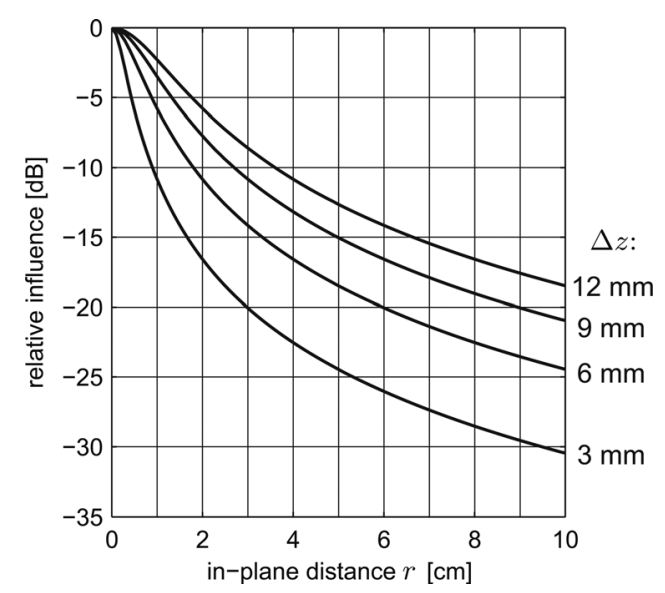

FIG. 2. Influence of a point in the source plane on a point in the measurement plane depending on the in-plane distance $r$ for several values of the measurement distance $\Delta z$. The plots are normalized with respect to the value at the closest point $(r=0)$. 


$$
P(x, y, z, \omega) \approx \frac{j \omega \rho e^{-j k \Delta z}}{2 \pi \Delta z} V_{z}\left(x, y, z_{0}, \omega\right) .
$$

This simplification leads to a direct relation between the measured pressure in the extreme near-field and the normal velocity of the closest point on the vibrating plate: Eq. (6) establishes a pointwise correspondence between the measurement plane and the reconstruction plane. This straightforward approach can be sufficient to retrieve the desired information on the vibration of the plate from the measurement.

The approximation can be analyzed by comparing Eq. (6) to the operator for radiation from a plate in the wavenumber-frequency domain as given by Williams: ${ }^{9}$

$$
\tilde{P}\left(k_{x}, k_{y}, z, \omega\right)=\frac{\omega \rho}{k_{z}} e^{-j k_{z} \Delta z} \tilde{V}_{z}\left(k_{x}, k_{y}, z_{0}, \omega\right),
$$

with

$$
k_{z}=\sqrt{k^{2}-k_{x}^{2}-k_{y}^{2}} .
$$

Here, $\tilde{P}\left(k_{x}, k_{y}, z, \omega\right)$ and $\tilde{V}_{z}\left(k_{x}, k_{y}, z_{0}, \omega\right)$ are the two-dimensional spatial Fourier transforms of $P(x, y, z, \omega)$ and $V_{z}\left(x, y, z_{0}, \omega\right)$, respectively:

$$
\tilde{P}\left(k_{x}, k_{y}\right)=\int_{-\infty}^{\infty} \int_{-\infty}^{\infty} P(x, y) e^{j\left(k_{x} x+k_{y} y\right)} \mathrm{d} x \mathrm{~d} y .
$$

The link between planar near-field acoustic holography and the approximation given by Eq. (6) can be established using Weyl's integral: ${ }^{10}$

$$
\frac{e^{-j k r}}{r}=\frac{-j}{2 \pi} \int_{-\infty}^{\infty} \int_{-\infty}^{\infty} \frac{e^{-j\left(k_{x} x+k_{y} y+k_{z} \Delta z\right)}}{k_{z}} \mathrm{~d} k_{x} \mathrm{~d} k_{y} .
$$

By setting $x=0$ and $y=0$, the following relation is obtained:

$$
\frac{j}{2 \pi} \frac{e^{-j k \Delta z}}{\Delta z}=\frac{1}{4 \pi^{2}} \int_{-\infty}^{\infty} \int_{-\infty}^{\infty} \frac{e^{-j k_{z} \Delta z}}{k_{z}} \mathrm{~d} k_{x} \mathrm{~d} k_{y}
$$

It can be seen that this expression provides the connection between Eq. (6) and Eq. (7). Therefore, the proposed approximation can be interpreted as averaging of the wavenumber spectrum in the spatial wavenumber domain as expressed by the right-hand side of Eq. (11). The wavenumber domain provides a plane wave decomposition of the acoustical field. Whereas for planar NAH, plane waves are assumed to propagate from the source plane to the measurement plane under all possible angles, the approximation given by Eq. (6) restricts the propagation of waves to the direction normal to the planes.

It is clear that this approximation cannot be used for accurate reconstruction of the normal velocity from the pressure measurement. For the qualitative analysis of the wave field, however, the perfect reconstruction of the normal velocity is not necessarily required. Typical applications include the retrieval of the dispersion relation and the detection of sources, reflectors and scatterers. In these cases, the desired information can directly be extracted from the pressure measurement using the simple inverse of Eq. (6):

$$
V_{z}\left(x, y, z_{0}, \omega\right) \approx \frac{2 \pi \Delta z}{j \omega \rho} e^{j k \Delta z} P(x, y, z, \omega) .
$$

Hence, an estimate of the surface normal velocity can be obtained without the inversion of a convolution operator as demanded by Eq. (1). However, since $P(x, y, z, \omega)$ and $V_{z}\left(x, y, z_{0}, \omega\right)$ are related by the frequency $\omega$, care must be taken in order to avoid the excessive amplification of noise at low frequencies when reconstructing the normal velocity using Eq. (12). In Sec. III, some typical applications of this approach are presented.

\section{B. Flexural waves}

The transverse motion of wave fields in plates can be described by the biharmonic equation for flexural waves: ${ }^{1}$

$$
\left(\nabla^{4}-k_{\mathrm{B}}^{4}\right) u=0
$$

with $u$ being the normal displacement and $k_{\mathrm{B}}$ being the flexural wavenumber depending nonlinearly on the frequency as shown by the dispersion relation:

$$
k_{\mathrm{B}}=\frac{\omega}{c(\omega)}=\frac{\sqrt{\omega}}{A} .
$$

Here, $A$ is the so-called dispersion constant:

$$
A=\sqrt[4]{\frac{h^{2} E}{12 \rho\left(1-\nu^{2}\right)}},
$$

with $h$ representing the plate thickness, $E$ being the Young's modulus, $\rho$ being the mass density, and $\nu$ representing the Poisson constant of the plate material.

The phase velocity $c$ for flexural waves depends on the frequency $\omega$ :

$$
c(\omega)=A \sqrt{\omega} .
$$

In a dispersive medium, a wavefront caused by an impulsive source signal tends to spread out during propagation. This leads to decreasing resolution with increasing distance from the source. In order to cope with this effect, it is first of all necessary to determine the dispersion characteristics.

\section{Retrieval of the dispersion relation}

The dispersion properties of a wave field can be determined by sampling the wave field in both space and time $(x, y, t)$. Application of Fourier transforms to both the temporal and the spatial dimensions leads to a representation in the wavenumber-frequency $\left(k_{\mathrm{x}}, k_{\mathrm{y}}, \omega\right)$ domain. Here, $k_{\mathrm{x}}$ and $k_{\mathrm{y}}$ are the spatial Fourier variables, and $\omega$ is the Fourier variable associated with time.

Transformation of Eq. (13) to the wavenumber-frequency domain leads to the following condition for plane propagating waves: 


$$
k_{\mathrm{B}}^{2}=k_{r}^{2}=k_{x}^{2}+k_{y}^{2} .
$$

Note that $k_{\mathrm{B}}$ is used to designate the wavenumber emanating from the wave equation, whereas $k_{r}$ represents a Fourier variable with respect to the radial spatial direction. For propagating plane waves, these two variables are equal. Hence, the flexural wavenumber $k_{\mathrm{B}}$ and the dispersion relation can be determined from a wavenumber-frequency representation of a wave field containing propagating waves. ${ }^{11}$

The dispersion relation for flexural waves is given by a parabolic curve described by Eq. (14). For acoustical waves, however, the relation between wavenumber and frequency is linear. Both curves are shown in Fig. 3 for a plate of $4 \mathrm{~mm}$ medium density fiberboard (MDF) as used for the experiments in Sec. III further below.

The acoustical radiation is known to be caused by the flexural motion of the plate. The air surrounding the plate can thereby be regarded as a spectral filter: only components of the flexural field within the shaded region in Fig. 3 cause radiation into the far-field. At the coincidence frequency, the phase velocities of flexural waves in the plate and longitudinal waves in air are equal. Flexural waves below the coincidence frequency create evanescent acoustical waves, which do not radiate into the far-field and can only be measured at a distance of typically less than a wavelength from the plate.

For the analysis of the vibrations in the plate, only the flexural wave field is of interest. Therefore, it is desirable to minimize or even remove the effect of propagation from the plate to the receiver. Near-field acoustic holography, on the one hand, tries to invert entirely the process of propagation through air. The vibrational field causing the measured radiation is thereby retrieved. As explained above, this method is based on the inversion of the integral operator in Eq. (1), which can be carried out either in the spatial domain or in the wavenumber domain, leading to an ill-posed problem requiring regularization in both cases.

The approach presented in this paper, on the other hand, suggests to change the physical measurement in such a way that the simple, approximate relation between measured

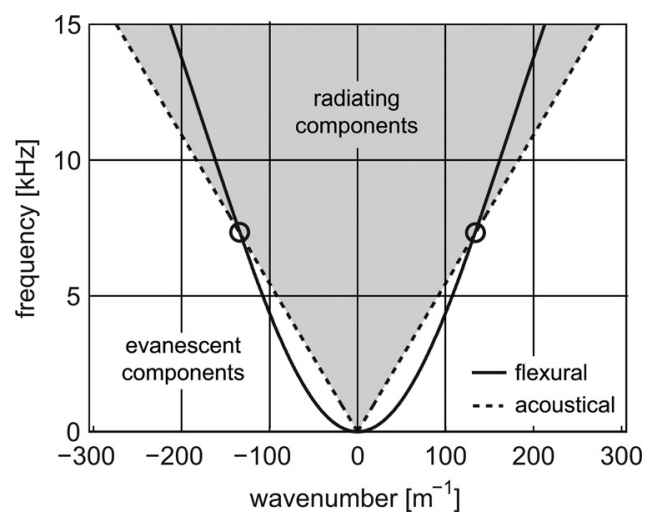

FIG. 3. Dispersion curves for flexural waves in a medium density fiberboard (MDF) plate of $4 \mathrm{~mm}$ thickness as used for the experiments in Sec. III and for acoustical waves in air at room temperature; the shaded region contains the components that are radiated into the far-field; evanescent components can be measured only close to the plate. The coincidence frequency is indicated by circles. pressure and estimated surface normal velocity described by Eq. (12) can be applied. It is clear that the correctness of the reconstruction obtained from this method depends on the degree to which the requirement of proximity between plate and receiver is fulfilled. The effects of propagation not compensated for contribute as 'noise' to the result. Due to the causal relation between flexural waves and acoustical radiation, it has to be expected that this 'noise' is correlated with the desired information.

\section{Dispersion compensation}

If the dispersion characteristics of the medium under investigation are known, it is possible to compensate for the effects of dispersion. Methods for dispersion compensation are well-known in the fields of optics ${ }^{12}$ and non-destructive material inspection. ${ }^{7}$ The result of this procedure does no longer represent a flexural wave field. Instead, an analogous wave field occurring in a non-dispersive medium is obtained. This enables the observation of wavefronts, thereby helping the interpretation of the wave field. Scatterers and reflectors that are difficult to recognize in the measured wave field can easily be detected in the dispersion-free representation.

A common approach for dispersion compensation is given by transforming the measured time signal to a spatial function of source-to-receiver distance. This can be achieved by means of a mapping from frequency to wavenumber. A plane flexural wave $U(\omega)$ propagating in one dimension is chosen for the derivation. The phase spectrum of a propagating flexural wave depends nonlinearly on the frequency:

$$
U(\omega)=S(\omega) e^{-j[\omega / c(\omega)] r_{0}}=S(\omega) e^{-j(\sqrt{\omega} / A) r_{0}},
$$

with $S(\omega)$ being the signal spectrum and $r_{0}$ being the distance between the source position and the receiver position.

The dispersive temporal signal can be obtained by application of an inverse Fourier transform:

$$
u(t)=\frac{1}{2 \pi} \int_{-\infty}^{\infty} S(\omega) e^{-j[\omega / c(\omega)] r_{0}} e^{j \omega t} \mathrm{~d} \omega .
$$

By performing a mapping from frequency $\omega$ to wavenumber $k_{\mathrm{B}}$ using the dispersion relation given by Eq. (14), the phase spectrum can be made linearly dependent on the Fourier variable. It is seen from Eq. (17) that the wavenumber $k_{\mathrm{B}}$ can be replaced by a spatial Fourier variable $k_{r}$ for plane propagating waves. Substitution of the integration variable $\omega$ by $k_{r}$ leads to the following equation:

$$
u(r)=\frac{1}{2 \pi} \int_{-\infty}^{\infty} S\left[k_{r}(\omega)\right] e^{-j k_{r} r_{0}} e^{j k_{r} r} \frac{\partial \omega}{\partial k_{r}} \mathrm{~d} k_{r} .
$$

In this equation, the frequency spectrum of the received signal is interpreted as a wavenumber spectrum by means of the mapping described by Eq. (14). This mapping has consequences for the result of the integral, too. Instead of being caused by a certain time of flight between source and receiver, events in the received signal are now related to a source-to-receiver distance expressed in terms of the spatial 
wavenumber spectrum $S\left(k_{r}\right)$. Therefore, the temporal signal on the left-hand side of Eq. (19) is replaced by a spatial signal $u(r)$ at the left-hand side of Eq. (20). However, the mapping itself has to be carried out from the temporal frequency to the spatial wavenumber domain due to the phase velocity being frequency-dependent. This processing step has to be applied to every receiver signal.

The inverse Fourier transform described by Eq. (20) results in a spatial signal containing a wavelet at the position corresponding to the distance $r_{0}$ between source and receiver:

$$
\begin{aligned}
u(r) & =\delta\left(r-r_{0}\right) *\left\{\frac{1}{2 \pi} \int_{-\infty}^{\infty} S\left[k_{r}(\omega)\right] \frac{\partial \omega}{\partial k_{r}} e^{j k_{r} r} \mathrm{~d} k_{r}\right\} \\
& =\delta\left(r-r_{0}\right) * s(r)
\end{aligned}
$$

with $\delta$ being the Dirac $\delta$ function, the asterisk denoting convolution, and $s(r)$ being the inverse spatial Fourier transform of $S\left[k_{r}(\omega)\right] \partial \omega / \partial k_{r}$. Dispersive temporal information is thus mapped to non-dispersive information on source-to-receiver distance. For every receiver, a spatial signal is obtained which provides a pseudo representation of the wave field as if it had occurred in a non-dispersive medium. The spectral correction factor $\partial \omega / \partial k_{r}$ ensures the conservation of spectral energy densities, but can be omitted from a signal processing point of view. ${ }^{13}$

This method for dispersion compensation is approximately valid for two-dimensional wave fields, too. In the far-field, a point source can be approximated by a combination of plane waves. The main difference to the above derivation is given by the distance-dependent attenuation factor $\sqrt{r_{0}}$ for point sources, which has to be taken into account. Therefore, the resulting wave field after dispersion compensation decays accordingly.

Figure 4 illustrates the idea of dispersion compensation by spectral mapping. It can be seen that for a discrete implementation, interpolation of the spectrum is required.

\section{APPLICATION TO MEASUREMENTS}

The proposed approach was verified by application to measurements taken on a medium density fiberboard (MDF) plate of 50 by $80 \mathrm{~cm}$ with a thickness of $4 \mathrm{~mm}$. The plate was excited by means of a small shaker (Brüel \& Kjær type 4810) with a linear sweep excitation signal up to $15 \mathrm{kHz}$ with a duration of $1 \mathrm{~s}$. A deconvolution filter was applied to the measured signals in order to determine the impulse response. The radiated pressure is measured in a plane at a distance of $5 \mathrm{~mm}$ from the plate on a grid of 70 by $100 \mathrm{~cm}$ with a spacing of $1 \mathrm{~cm}$ using a 1/4 in. microphone (Brüel \& Kjær type 4136). Figure 5 shows the plate, the point of excitation, and two small wooden beams glued to the plate in order to create scattered and reflected flexural waves.

\section{A. Determination of the dispersion relation}

Figure 6 presents the measured pressure information in the wavenumber-frequency domain. A slice at $k_{y}=0$ is shown. It is known that the flexural wavenumber $k_{\mathrm{B}}$ of waves propagating in the plate and the in-plane component $k_{r}$ in the measurement plane above the plate are equal to each other due to continuity conditions at the plate surface. ${ }^{1}$ For the slice at $k_{y}=0$ shown in Fig. 6, Eq. (17) states that $k_{\mathrm{B}}=k_{x}$. Therefore, the dispersion relation for the flexural field can directly be retrieved from this slice of the pressure measurement in the wavenumber-frequency domain. The measurement is taken close enough to the plate to contain evanescent components, which reveal the dispersion relation of the flexural field below the coincidence frequency.

The dispersion relation can be determined by fitting a curve to the measurement depicted in Fig. 6. The phase velocity of the flexural waves in the plate is shown in Fig. 7; it is obtained from the measured dispersion relation by means of Eq. (14). This information can subsequently be used for

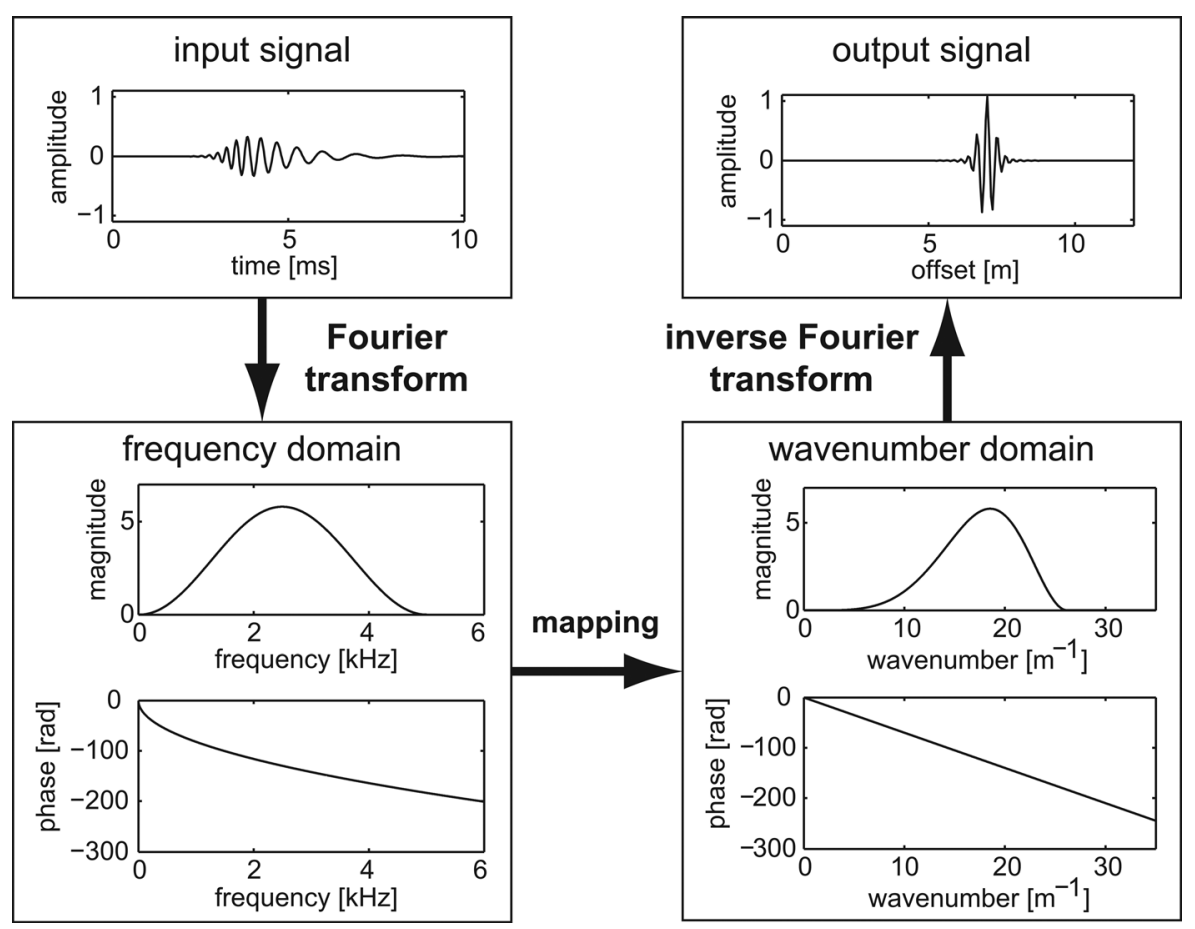

FIG. 4. Illustration of dispersion compensation by mapping from frequency to wavenumber. The phase spectrum is linearized, leading to a compact spatial output signal containing information about the distance between source and receiver (Ref. 13). 


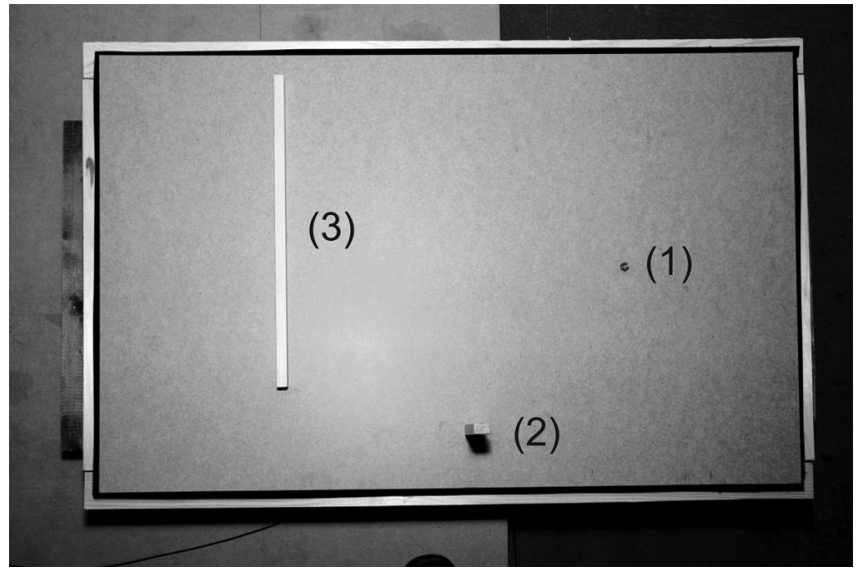

FIG. 5. MDF plate used for the experiments; the point of excitation (1) can be seen. Two small wooden beams are glued to the plate in order to achieve scattering (2) and reflection (3) of incident flexural waves.

dispersion compensation as described in Sec. II D and demonstrated in the following section.

\section{B. Visualization of the wave field}

An approximate reconstruction of the normal velocity obtained by Eq. (12) is shown in Fig. 8(a). Due to the effects of dispersion, there are no clear wavefronts visible. Figure 8(b) shows a reconstruction of the normal velocity of the plate obtained by near-field acoustic holography, which is used for comparison with the proposed approach. The NAH result is obtained by inversion of the Rayleigh integral given by Eq. (1). To this end, the forward and adjoint operators of the operation described by the Rayleigh integral are formed. A conjugate gradient scheme is then used to obtain an estimate of the normal velocity. The inversion is carried out in the spatial domain in order to avoid truncation effects and spectral leakage. $^{14}$

Ideally, the normal velocity outside the unbaffled plate should be several orders of magnitude smaller than the normal velocity on the plate. ${ }^{15}$ However, it can be observed in Fig. 8(b) that there are significant wave field components in the recon-

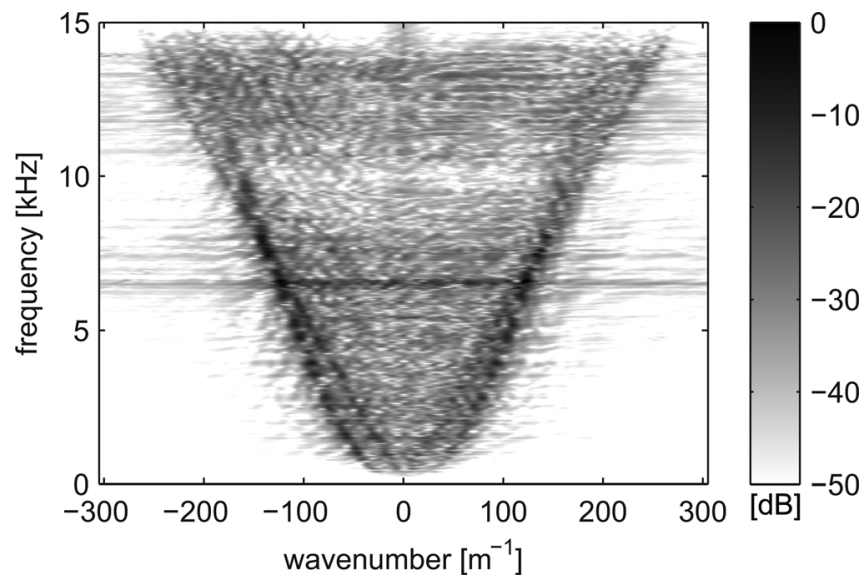

FIG. 6. Wavenumber-frequency domain representation of a pressure measurement taken close to a vibrating plate made of $4 \mathrm{~mm} \mathrm{MDF}$; the field shows both the acoustical and the flexural dispersion relation; compare to Fig. 3 .

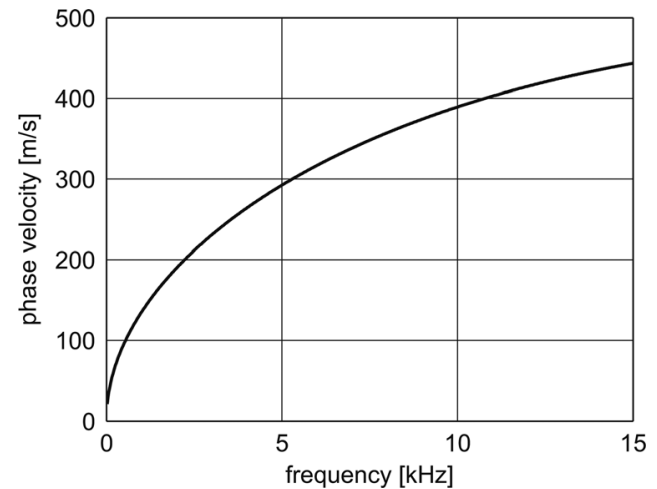

FIG. 7. Phase velocity of flexural waves in a $4 \mathrm{~mm}$ MDF plate as retrieved from the measurement shown in Fig. 6.

structed velocity outside the plate, i.e., within a region of $10 \mathrm{~cm}$ width surrounding the plate. This is due to several reasons. First of all, the reconstruction of the surface normal velocity is imperfect since the measurement distance of $5 \mathrm{~mm}$ can only be kept approximately for the entire plate. Furthermore, there are waves which are radiated from the far side of the plate and reach the microphone after being reflected from the floor of the semi-anechoic room used for the measurements.

In a subsequent step, the method for dispersion compensation presented in Sec. II D is applied to the reconstructed surface normal velocities obtained by application of Eq. (12) and by NAH. For optimal resolution of the wave fronts in a visual representation, the spectrum of the wave field should be approximately flat. Therefore, the spectra of both reconstructions are adjusted equally by application of a filter aiming for flat amplitude spectra.

The results are shown in Fig. 8(c), for the approximate reconstruction of the normal velocity according to Eq. (12), and in Fig. 8(d), for the NAH reconstruction. It can be seen that the visualizations of both approaches do not differ too much after dispersion removal. In both cases, it is possible to see the reflections from the plate boundaries and to identify the circular wavefront caused by the scatterer on the plate.

Figure 9 shows the same visualizations as Fig. 8 at a later point in time. The effects of dispersion are worse, and compensation is required even more in order to be able to analyze the observed wave field. Once more, the additional effort of inverting the Rayleigh integral for the reconstruction of the surface normal velocity does not provide information that cannot be obtained from the pressure measurement by application of the approximate point-to-point relation given by Eq. (12). In both Figs. 9(c) and 9(d), the reflection from the wooden beam glued to the plate can be observed.

\section{DISCUSSION}

It has been shown that an approximate reconstruction of the normal velocity based on pressure measurements taken in the extreme near-field can be used for the visualization of the wave field in the plate. It enables the detection of transmission paths and inhomogeneities. In this context, the approximation for the reconstruction of the surface normal velocity proposed in this work and the classical method of near-field acoustic holography have been shown to exhibit 

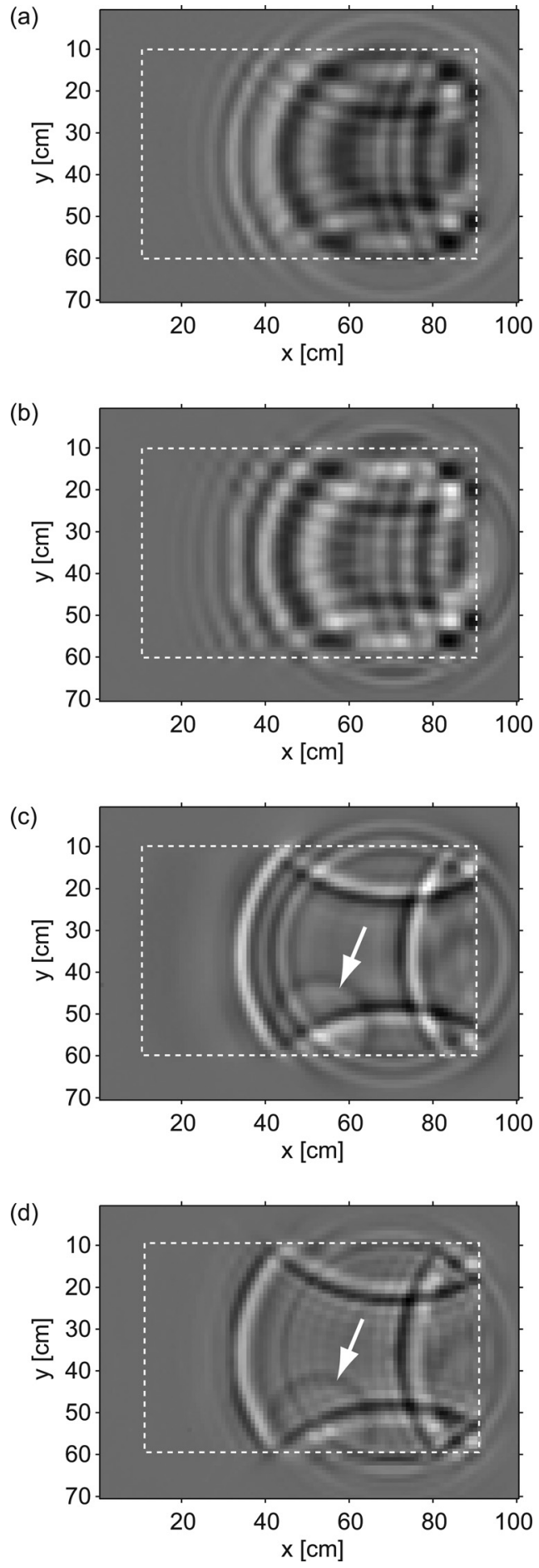

FIG. 8. Visualization of the wave field at $t=0.8 \mathrm{~ms}$ : (a) estimate of the normal velocity obtained according to Eq. (12), (b) reconstructed normal velocity obtained by NAH, (c) dispersion-compensated representation of the field shown in panel (a), (d) dispersion-compensated representation of the field shown in panel (b); the arrow indicates a circular wavefront caused by the scattering object (2) in Fig. 5. All amplitudes are normalized. The boundary of the plate is indicated by the dashed line.

similar performance in the extreme near-field. The application of dispersion compensation, however, has a significant positive effect on the resolution of the visualization of the wave field.

A drawback of the presented approach for the reconstruction of the normal velocity is given by the fact that the
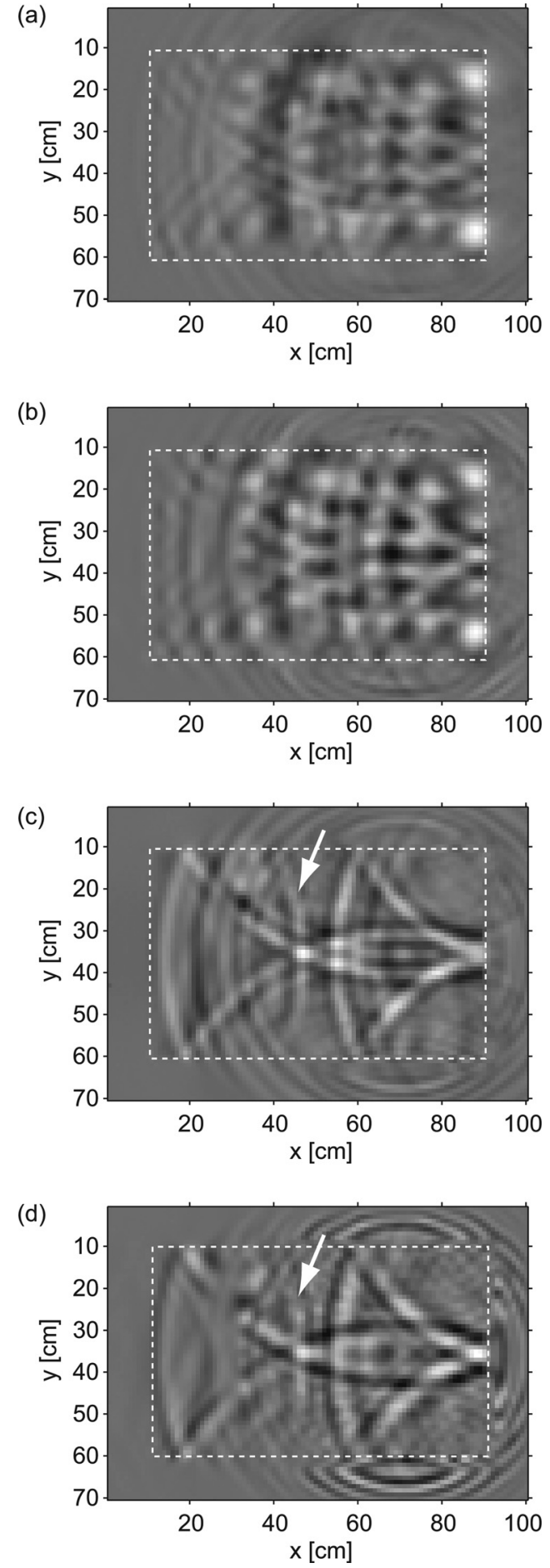

FIG. 9. Visualization of the wave field at $t=1.3 \mathrm{~ms}$ : (a) estimate of the normal velocity obtained according to Eq. (12), (b) reconstructed normal velocity obtained by NAH, (c) dispersion-compensated representation of the field shown in panel (a), (d) dispersion-compensated representation of the field shown in panel (b); the arrow indicates a wavefront caused by the reflecting object (3) in Fig. 5. All amplitudes are normalized. The boundary of the plate is indicated by the dashed line.

pressure measurement contains information which is determined by a small region on the vibrating plate rather than by a point. The result of the point-to-point relation given by Eq. (12) is, therefore, smoothed in comparison to measurements obtained by laser vibrometry or NAH. The disadvantages of laser vibrometry are the higher hardware costs and the 
requirement of special surface conditions. Near-field acoustic holography does not suffer from these drawbacks to the same extent. However, it requires extensive processing of the measurements in order to focus the measured information on the plane of interest.

For the simple approach presented in this paper, the analysis of the quantitative error and the limit of validity are proposed as a topic for further research. Furthermore, better focusing could be achieved by changing the directivity of the receiver. For instance, the usage of a velocity probe instead of an omnidirectional microphone is expected to lower the susceptibility to waves radiated from surrounding points on the plate. The achievable resolution could thereby be improved, and the presence of artifacts reduced. However, the present paper focuses on the quality of results that can be achieved even with the simple approach based on measurements with an omnidirectional microphone. The usage of a velocity probe is, therefore, suggested for future research.

\section{CONCLUSIONS}

An approximate method for the reconstruction of the surface normal velocity from near-field pressure measurements has been proposed as an alternative to near-field acoustical holography. For measurements taken in the extreme near-field of a vibrating plate, it could be shown that the effort of acoustical holography is not necessarily required to obtain a useful reconstruction of the normal velocity. Furthermore, qualitative information, such as the propagation speed in the plate, can be determined directly from the pressure measurements.

For the analysis of flexural waves, dispersion compensation based on a mapping from frequency to wavenumber can help to increase the resolution of visualizations of the wave field and enable the visibility of propagating wavefronts. The detection of scattering and reflecting inhomogeneities in the plate is thereby simplified.

The usage of a velocity probe instead of a pressure-sensitive microphone is suggested for future research since it is expected to improve the achievable resolution further.

\section{ACKNOWLEDGMENTS}

The author would like to thank Diemer de Vries for his support with the research carried out. This research is supported by the Dutch Technology Foundation STW (Stichting voor de Technische Wetenschappen), which is the applied science division of NWO (Netherlands Organization for Scientific Research), and the Technology Programme of the Ministry of Economic Affairs.

${ }^{1}$ L. Cremer, M. Heckl, and B. A. T. Petersson, Structure-Borne Sound: Structural Vibrations and Sound Radiation at Audio Frequencies, 3rd ed. (Springer, New York, 2005), pp. 483-485.

${ }^{2}$ J. D. Maynard, E. G. Williams, and Y. Lee, "Nearfield acoustic holography: I. Theory of generalized holography and the development of NAH," J. Acoust. Soc. Am. 78, 1395-1413 (1985).

${ }^{3}$ F. Jacobsen and Y. Liu, "Near field acoustic holography with particle velocity transducers," J. Acoust. Soc. Am. 118, 3139-3144 (2005).

${ }^{4}$ E. G. Williams, "Regularization methods for near-field acoustical holography,” J. Acoust. Soc. Am. 110, 1976-1988 (2001).

${ }^{5}$ B. K. Kim and J. G. Ih, "Design of an optimal wave-vector filter for enhancing the resolution of reconstructed source field by near-field acoustical holography (NAH)," J. Acoust. Soc. Am. 107, 3289-3297 (2000).

${ }^{6} \mathrm{~N}$. Valdivia and E. G. Williams, "Krylov subspace iterative methods for boundary element method based near-field acoustic holography," J. Acoust. Soc. Am. 117, 711-724 (2004).

${ }^{7} \mathrm{P}$. D. Wilcox, "A rapid signal processing technique to remove the effect of dispersion from guided wave signals," Ferroelectr. Freq. Control 50, 419-427 (2003).

${ }^{8}$ F. Fahy, Sound and Structural Vibration: Radiation, Transmission and Response (Academic, London) (1985), pp. 56-57.

${ }^{9}$ E. G. Williams, Fourier Acoustics (Academic, London, 1999), pp. 90-91.

${ }^{10} \mathrm{M}$. Tygel and P. Hubral, "Transient representation of die SommerfeldWeyl integral with application to the point source response from a planar acoustic interface," Geophysics 49, 1495-1505 (1984).

${ }^{11}$ D. Alleyne and P. Cawley, "A two-dimensional Fourier transform method for the measurement of propagating multimode signals," J. Acoust. Soc. Am. 89, 1159-1168 (1991).

${ }^{12} \mathrm{E}$. Brinkmeyer and R. Ulrich, "High-resolution OCDR in dispersive waveguides," Electron. Lett. 26, 413-414 (1990).

${ }^{13}$ L. Hörchens, "Imaging of material inhomogeneities with flexural waves," Ph.D. dissertaion, Delft University of Technology, The Netherlands, 2010.

${ }^{14}$ Y.-C. Chao, "An implicit least-square method for the inverse problem of acoustic radiation," J. Acoust. Soc. Am. 81, 1288-1292 (1987).

${ }^{15} \mathrm{E}$. G. Williams, "Numerical evaluation of the radiation from unbaffled, finite plates using the FFT," J. Acoust. Soc. Am. 74, 343-347 (1983). 